\title{
Le applicazioni mobili in Sanità: aspetti regolatori e review sul loro utilizzo nelle patologie croniche più frequenti
}

\author{
Marco Spissu ${ }^{1}$, Maura Fiamma ${ }^{2}$, Pina Meloni ${ }^{3}$, Antonio Nicoletti ${ }^{4}$, Alessandro Carrus ${ }^{5}$, Sara Sanna ${ }^{5}$, Francesco Logias ${ }^{5}$ \\ ${ }^{1}$ Area P.C.C., ATS. Sardegna, ASSL Olbia, Olbia \\ 2 U.O.C. Laboratorio Analisi, P.O. San Francesco, ATS. Sardegna, ASSL Nuoro, Nuoro \\ ${ }^{3}$ U.O.S. Cardiologia, P.O. C. Zonchello, ATS. Sardegna, ASSL Nuoro, Nuoro \\ ${ }^{4}$ U.O.S. Nefrologia e Dialisi, P.O. Ospedale Civile, AST Cosenza, Cosenza \\ ${ }^{5}$ U.O.C. Nefrologia e Dialisi, P.O. San Francesco, ATS. Sardegna, ASSL Nuoro, Nuoro
}

\begin{abstract}
Mobile apps in healthcare: regulations and review of their use in the treatment of frequent chronic diseases Today, mobile devices, smartphones and tablets have become a fundamental part of our lives, revolutionizing much of their aspects. The combination of a very powerful hardware and the presence of many sensors and instant connectivity with the huge availability of applications for every need, including those about health and healthcare, make mobile devices a powerful tool for the growing and the development of the "digital healthcare". In fact major competitors in medical and healthcare market are starting to look at the "digital healthcare" and its revenues with great interest.

Despite there is a tight control on what is classified as a medical device in the strict sense, today there is not yet clarity on all software applications categorized in health and wellness, but which are not classified strictly as medical devices and which are therefore not subjected to the regulation of medical devices.
\end{abstract}

Keywords: Healthcare, Medical devices, Mobile Apps, Mobile health

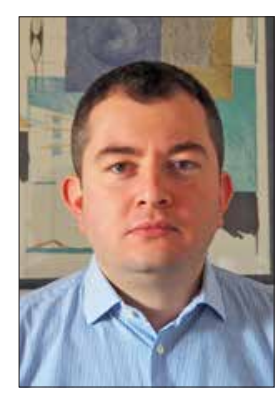

Marco Spissu

\section{Introduzione}

I cosiddetti "dispositivi mobili", smartphone e tablet, sono diventati parte integrante della nostra vita, rivoluzionandone gran parte degli aspetti. Di fatto, ognuno di noi ne possiede almeno uno e li possiamo facilmente considerare uno degli oggetti da cui risulta quasi impossibile separarsi, grazie alla possibilità di eseguire tramite un unico device una serie di compiti spesso com-

Accepted: January 12, 2017

Published online: February 14, 2017

Indirizzo per la corrispondenza:

Dr. Marco Spissu

Area P.C.C., ATS. Sardegna, ASSL Olbia

Via Bazzoni-Sircana, 2

07026 Olbia

marco.spissu@gmail.com plessi, per cui prima erano necessari diversi dispositivi: telefono, PC, PDA, fotocamera, strumenti di misura, GPS e così via. Grazie alla veloce progressione tecnologica degli ultimi anni, i dispositivi mobili attuali sono dotati di un hardware che può facilmente competere con quanto installato nei PC di dieci anni fa: processori multi-core performanti, grande capacità di memorizzazione dei dati (spesso ampliabile con costi ridotti) e varie tipologie di sensori multifunzionali di cui sono dotati. Se a tali caratteristiche ne aggiungiamo altre come l'elevata portabilità, la connettività istantanea, la possibilità di personalizzazione e il rilevamento della posizione ecco spiegato il loro enorme successo e la grande distribuzione in tutto il Mondo: si stima, infatti, che entro il 2020 gli utenti connessi a dispositivi mobili nel mondo saranno 5 miliardi e mezzo, ovvero più o meno il $70 \%$ della popolazione mondiale, e ciò significa che ci saranno più persone con un cellulare (5.4 miliardi) di quelle con l'accesso all'energia elettrica (5.3 miliardi).

Tutte le migliori caratteristiche tecniche succitate però non sono sufficienti per spiegare, per esempio, l'enorme successo degli smartphone, sempre più considerati come sistemi portatili per l'elaborazione dei dati piuttosto che come 


\section{Categorie Applicazioni mHealth (2015)}

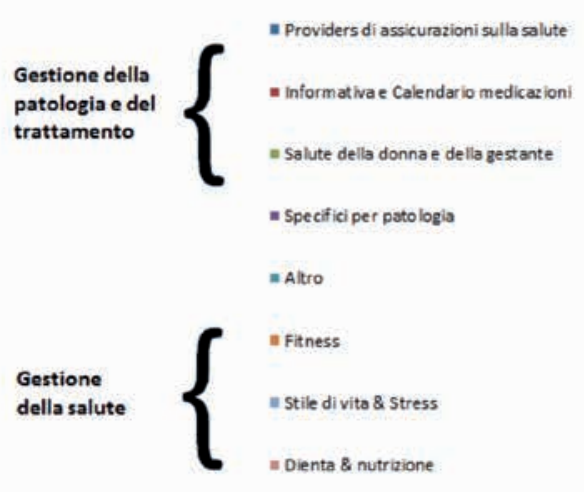

Fig. 1 - Distribuzione percentuale delle tipologie di App sanitarie - IMS 2015. semplici dispositivi di comunicazione e, infatti, spesso li si utilizza più per navigare sul web, controllare la posta, scattare foto e aggiornare il nostro status sui social piuttosto che per effettuare chiamate e inviare semplici SMS. II loro grande successo è dovuto soprattutto alle innumerevoli applicazioni mobili o "App" disponibili. Una App è un software specifico che può essere scaricato e utilizzato tramite il proprio dispositivo mobile, per eseguire determinati compiti.

Il grande successo e la popolarità delle App è dovuto alla loro semplicità di utilizzo, alla grafica accattivante delle loro interfacce, alla velocità e, soprattutto, alla loro portabilità: rendono disponibili le informazioni richieste e a portata di mano per ogni utente, ovunque e in qualsiasi momento. Le applicazioni mobili abbracciano i più svariati settori tra commercio, intrattenimento, pubblicità e istruzione e stanno prendendo sempre più piede anche nel campo della salute e dell'assistenza sanitaria, dove un numero sempre crescente di applicazioni è in via di sviluppo, tanto che i maggiori negozi digitali hanno istituito categorie a loro dedicate: "Wellness", "Health", "Healthcare" e così via. Si può tranquillamente affermare che tali applicazioni stanno iniziando a rivoluzionare il modo in cui si usufruisce dei servizi sanitari in tutto il mondo e il modo in cui si "fa Sanità" in molti ambiti specialistici e non. Anche la Sanità, quindi, sta muovendo i suoi passi a grande velocità nella direzione in cui i servizi sono offerti agli utenti (i pazienti) tramite tramite i loro stessi dispositivi di uso comune (smartphone, tablet, etc.), portando alla nascita, negli ultimi anni, della cosiddetta " $m \mathrm{He}$ alth", ovvero la "sanità mobile". La cosiddetta mHealth è la "pratica della medicina e della sanità pubblica supportata da dispositivi mobili, quali telefoni cellulari, dispositivi per il monitoraggio dei pazienti, computer palmari e altri dispositivi senza fili".

Rientrano all'interno della mHealth le App per il benessere e lo stile di vita che possono connettersi a dispositivi medici o sensori (per esempio, braccialetti od orologi), gli strumenti di comunicazione (SMS, e-mail, ecc.) con informazioni sanitarie e promemoria dei farmaci da assumere (informazione e motivazione), i consigli dietetici e per restare in forma, i sistemi di consulenza personalizzata e la telemedicina attraverso comunicazioni senza fili. Inoltre in essa rientrano tutte le varie soluzioni tecnologiche che permettono, tra le altre cose, di misurare parametri vitali come la frequenza cardiaca, il livello di glicemia, la pressione sanguigna, la temperatura corporea e l'attività cerebrale.

Secondo un rapporto pubblicato dall'IMS Institute of Healthcare Informatics, nell'anno 2015 erano già disponibili circa 165.000 applicazioni mobili appartenenti alla macroarea "sanitaria" (2). Interessante da notare il trend di distribuzione delle App sanitarie studiate nel 2015: tra le App di " $m$ Health" censite, circa il $70 \%$ è destinato al segmento di mercato del benessere e della forma fisica, mentre il 30\% è destinato agli operatori sanitari e facilita l'accesso ai dati dei pazienti, il teleconsulto e il monitoraggio dei parametri dei pazienti, la diagnostica per immagini, l'accesso a informazioni su prodotti farmaceutici e così via (Fig. 1).

Se il trend di crescita sarà rispettato, si stima che il settore garantirà a breve un enorme giro d'affari per le aziende che operano nel settore medicale: già nel 2017 si prevedono, infatti, introiti attorno ai 23 miliardi di dollari, di cui 6.9 costituiti dal mercato europeo.

È chiaro, quindi, che se da una parte la mHealth può essere un'opportunità per migliorare l'assistenza sanitaria, migliorandone la qualità e l'efficienza, dall'altra è indubbio l'interesse che questo settore suscita anche da un punto di vista economico.

Com'è facile intuire, l'insieme delle tecnologie legate alla $m$ Health ha enormi potenzialità migliorative per la salute individuale e la salute pubblica. Tra i principali fattori positivi 
di queste nuove tecnologie che si stanno già rapidamente diffondendo nella nostra società, si richiamano:

- la promozione di uno stile di vita salutare, il miglioramento della consapevolezza e la partecipazione attiva e la motivazione degli individui alla salute;

- il miglioramento tramite la facilitazione e la velocizzazione della comunicazione medico-paziente, la personalizzazione di trattamenti e l'incremento dell'autonomia e della sicurezza del paziente che può essere controllato e localizzato a distanza;

- il miglioramento dell'efficienza del sistema sanitario, attraverso la riduzione dei costi dell'assistenza e dell'ospedalizzazione, l'analisi mediante l'uso del monitoraggio a distanza o della telemedicina, la velocizzazione e il miglioramento quantitativo e qualitativo della comunicazione di informazioni al paziente da parte della struttura sanitaria;

- il contributo alla ricerca attraverso la facilitazione della raccolta di dati individuali e ambientali, utili per il singolo e per la collettività (soprattutto ricerche epidemiologiche, studio della correlazione tra determinate condizioni mediche e ambientali, ecc.);

- l'ampliamento dell'accesso alle cure, con la possibilità di raggiungere utenti che altrimenti non avrebbero l'assistenza medica;

- lo stimolo alla ricerca, alla produzione e all'innovazione, anche attraverso la costituzione di start-up dedicate;

- la facilitazione della possibilità di condivisione di casi clinici e la richiesta di secondi pareri in real-time per i medici.

A fronte di tali fattori positivi e di straordinaria importanza per il progresso scientifico e tecnologico, è necessario però evidenziare anche alcune problematiche emergenti, su cui è necessario riflettere, con l'obiettivo di rendere l'ambito della $m$ Health compatibile con la tutela dei diritti, della sicurezza e del benessere degli utenti.

Il primo aspetto fondamentale da affrontare è quello riguardante la tutela della salute e della sicurezza dell'utente, ovvero capire in che modo queste siano influenzate dalla tecnologia utilizzata. In sostanza si tratta di determinare i criteri per la distinzione tra le tecnologie legate alla $\mathrm{mHe}$ alth che rientrano tra i dispositivi medici e quelle che invece non vi rientrano. Tale distinzione è rilevante in quanto nel primo caso le applicazioni sulla salute prima di essere autorizzate sul mercato devono essere conformi alle normative europee vigenti e ai requisiti di certificazione: le normative disciplinano come si devono usare i dispositivi, chi li può usare e quali sono le applicazioni; la certificazione garantisce la sicurezza, nel senso della "non pericolosità", ma non l'efficacia. Nel secondo caso, ossia quando si esclude che le applicazioni per la salute siano dispositivi medici, non esiste una regolamentazione specifica, ma solo la generale tutela del consumatore.

\section{Le App come dispositivo medico: la Direttiva Europea dei Dispositivi Medici e gli Standard di settore}

Come detto, nel concetto di mHealth rientrano anche le App per il benessere e lo stile di vita che possono connettersi a dispositivi medici o sensori (per esempio, braccialetti od orologi), i sistemi di consulenza personalizzata, gli SMS con informazioni sanitarie e promemoria dei farmaci da assumere, la telemedicina e cosi via. La mHealth sta diventando, quindi, un argomento chiave e non a caso la Commissione Europea ha recentemente (2014) pubblicato un "LIBRO VERDE" sulla sanità mobile al fine di avviare una consultazione delle parti interessate sugli ostacoli esistenti e sulle questioni connesse alla diffusione della sanità mobile.

Ma quando una App può essere definita come Dispositivo Medico e quando, invece, contrariamente alle apparenze, non lo è?

Con la nuova Direttiva dei Dispositivi Medici della Comunità Europea 2007/47/EC, che aggiorna la precedente Direttiva 93/42/EC, è stata formalmente riconosciuta l'importanza del software nel settore dei dispositivi medici, nonché l'esistenza del software stesso come dispositivo medico anche in modo indipendente, ovvero, il cosiddetto software stand alone. La definizione riportata nella Direttiva definisce:

"dispositivo medico: (...) software (...) utilizzato da solo o in combinazione, compreso il software destinato dal Fabbricante a essere impiegato specificamente con finalità diagnostiche e/o terapeutiche e necessario al corretto funzionamento del dispositivo, destinato dal Fabbricante a essere impiegato sull'uomo a fini di:

- diagnosi, prevenzione, controllo, terapia o attenuazione di una malattia;

- diagnosi, controllo, terapia, attenuazione o compensazione di una ferita o di un handicap;

- studio, sostituzione o modifica dell'anatomia o di un processo fisiologico;

- intervento sul concepimento, la cui azione principale voluta nel o sul corpo umano non sia conseguita con mezzi farmacologici né immunologici né mediante metabolismo, ma la cui funzione possa essere assistita da questi mezzi".

Risulta chiaro, quindi, che il criterio cardine è la destinazione d'uso medicale e terapeutica di un prodotto (intended purpose), ovvero l'utilizzo al quale è destinato il dispositivo, coerentemente con le informazioni e dichiarazioni rese dal Produttore nell'etichettatura, nelle istruzioni per l'uso e nel materiale promozionale.

I criteri per classificare e categorizzare il software (e quindi le App) sono descritti nelle specifiche Linee Guida "MEDDEV 2.1/6", aggiornate nel Luglio 2016, nelle quali viene anche suggerito un diagramma decisionale per guidare alla qualificazione del software come dispositivo medico (Fig. 2). 


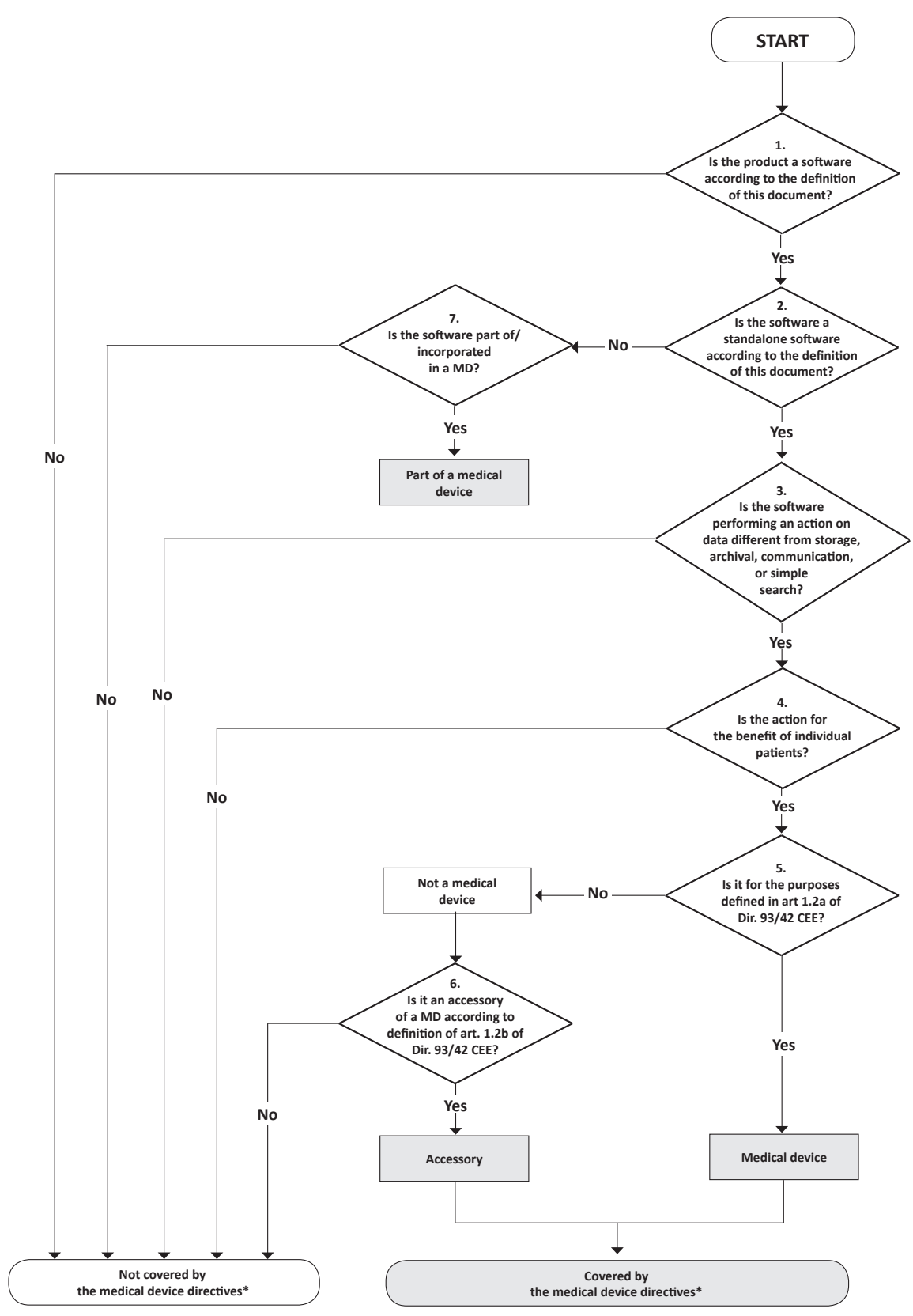

Fig. 2 - Diagramma decisionale - software classificabile come dispositivo medico.

TABELLA I - Contesto legislativo

\begin{tabular}{llll}
\hline & Codice & Descrizione & Campo di applicazione \\
\hline 1 & ISO 13485:2016 & Dispositivi medici - sistemi per la gestione della qualità & Fabbricanti dei Dispositivi Medici \\
2 & DIRETTIVA 93/42/CEE & Direttiva dei Dispositivi Medici (MDD) & \\
3 & DIRETTIVA CE 47/2007 & Medical Device Software - Software Life Cycle Processes & Dispositivi Medici \\
& & $\begin{array}{l}\text { Emendamento delle Dir. Eur. riguardanti i Dispositivi Medici } \\
\text { (inclusa la 93/42/CEE) }\end{array}$ & \\
5 & ISO IEC 62304:2007 & Medical Device Software - Software Life Cycle Processes & \\
\hline
\end{tabular}


Le Linee Guida "MEDDEV" hanno chiarito definitivamente che il software stand alone, anche quando sia utilizzato in ambiente medico con finalità generali, non è classificabile come un dispositivo medico. Quando invece esso è specificatamente destinato dal produttore a essere utilizzato per uno o più scopi medici, descritti nella definizione di dispositivo medico precedentemente riportata, si può classificare come dispositivo medico.

L'ossatura principale delle norme internazionali (Tab. I)che, nate per la regolamentazione del software medicale, regolamentano anche le App classificabili come dispositivo medico è:

- la EN ISO 13485:2016 - "Medical Devices - Quality Management Systems - Requirements for Regulatory Purposes", per quanto riguarda i sistemi per la gestione della qualità e quindi la convalida dei processi produttivi e dei software applicati per la produzione dei Dispositivi Medici;

- la EN IEC 62304:2007 - "Medical Device - Software life cycle processes", che assicura che un software di qualità sia prodotto mantenendo un processo di sviluppo definito e controllato attraverso l'accurata programmazione e lo svolgimento e il controllo meticoloso di tutte le fasi del cosiddetto ciclo di vita (pianificazione, progettazione, sviluppo, testing, manutenzione, ecc.), minimizzando i rischi per la salute ad esso correlati e che categorizza i software in tre classi, in base alla loro sicurezza;

- la ISO 14971:2012: relativa all'applicazione della valutazione del rischio ai dispositivi medici.

Ad esse, risulta importantissimo affiancare, tenendo presente il contesto di applicazione, anche la norma "EN IEC 80001:2010 - Application of risk management for IT-networks incorporating medical devices - Part 1: Roles, responsibilities and activities", relativa all'applicazione della gestione del rischio alle reti IT che includono dispositivi medici. Basti pensare a tutti i professionisti di settore (medici, personale sanitario, ecc.) che dispongono sia di dispositivi BYOD ("Bring your own device", ovvero i dispositivi personali usati sul luogo di lavoro per scopi produttivi) che di dispositivi COPE ("Corporate-owned personally enabled", ovvero i dispositivi aziendali utilizzati anche fuori dal contesto produttivo) con tutte le implicazioni relative.

Recentemente è stato pubblicato sul sito della " $D G$ Health and Consumer" della Comunità Europea l'upgrade del Manuale Borderline che contiene un capitolo ad hoc sui criteri di qualificazione delle App e dei software, dal quale si evince, per esempio, che non rientrano nella classificazione delle App e dei software come Dispositivo Medico quelli:

- che risultano essere copie elettroniche di testi e materiale medico non contenenti informazioni di specifici pazienti;

- per accedere e registrare e/o tracciare suggerimenti correlati allo stato generale di salute o benessere, ma non destinati a cure, trattamenti, diagnosi, ecc.;

- dedicati alla realizzazione della funzione di cartella clinica elettronica (EHR) o cartella clinica personale.
D’altra parte, invece, risultano essere classificabili come dispositivo medico le App:

- che risultano essere estensioni di dispositivi medici, con funzioni di controllo degli stessi o di visualizzazione, memorizzazione, analisi o trasmissione di dati provenienti dal dispositivo medico stesso;

- che trasformano una piattaforma mobile (smartphone o tablet) in un vero e proprio dispositivo medico, utilizzando accessori o sensori con funzioni da dispositivo medico;

- che utilizzano formule o algoritmi, trasformando informazioni derivanti da singoli pazienti (dati in input) in risultati o raccomandazioni utili alla diagnosi o al trattamento del paziente (output) e utilizzati in assistenza o per decisioni cliniche.

\section{II problema della privacy}

Con il repentino sviluppo della mHealth si pone sempre più impellente il problema della tutela dei dati sanitari e non, che vengono raccolti, conservati e qualche volta anche elaborati. Le problematiche riguardano non solo la tipologia e l'affidabilità dei dati, ma anche la correttezza del trattamento delle informazioni riservate raccolte per mezzo delle App installate nei dispositivi portatili. Questi sono potenzialmente in grado di raccogliere grandi quantità di informazioni (per esempio dati memorizzati sul dispositivo dall'utente e dati forniti da vari sensori, come l'ubicazione, la frequenza cardiaca, ecc.) e di trattarle, anche in paesi terzi al di fuori della Comunità Economica Europea, teoricamente al fine di fornire all'utente finale servizi nuovi e innovativi. A tale riguardo, secondo il gruppo consultivo dei Garanti europei "Article 29 Data Protection Working Party", sussistono gravi rischi per la privacy derivanti dall'uso delle App. Tali rischi sono resi ancora più gravi poiché, per esempio, la stretta interazione con $\mathrm{i}$ sistemi operativi rende possibile il fatto che alcune App sono in grado di raccogliere molti più dati di quelli raccolti tramite un browser Internet tradizionale. È stata inoltre rilevata una grande frammentazione degli operatori del settore (sviluppatori, titolari delle $A p p$, venditori, produttori di sistemi operativi, produttori di smartphone, ecc.) e ciò aggiunge ulteriori complicazioni. In sostanza, nel Parere dell'“Article 29", vengono identificati principalmente i seguenti problemi:

- mancanza di trasparenza, poiché spesso l'utente finale non riceve informazioni sufficienti relativamente a quali dati siano stati elaborati e da chi (nello studio è stato stimato che solo il $61.3 \%$ delle 150 App più scaricate dispone di una privacy policy);

- mancanza del libero consenso informato, poiché l'utente non è messo nella condizione di acconsentire in maniera libera e informata al trattamento dei propri dati personali tramite la App; 
- $\quad$ carenti misure di sicurezza, poiché i dati personali raccolti tramite la App spesso non sono trattati e conservati secondo adeguati standard di sicurezza;

- $\quad$ violazione del principio di limitazione delle finalità, visto che i dati personali spesso vengono utilizzati al di là degli scopi specifici per cui sono stati raccolti.

Uno studio del Financial Times ("Health apps run into privacy snags", 2013) ha rivelato che 9 delle 20 App più utilizzate in campo sanitario trasmettono i dati raccolti a una delle principali aziende di profilazione sul modo in cui la gente usa i telefoni cellulari. In generale, le informazioni trasmesse sono nella maggior parte dei casi dati personali, in quanto si riferiscono a persone fisiche direttamente o indirettamente identificate o identificabili.

In ultimo, desta legittime preoccupazioni anche la sicurezza sul trattamento dei dati relativi alla salute delle persone che utilizzano tecnologie $\mathrm{mHealth}$, dal momento che i loro dati personali potrebbero essere facilmente divulgati, accidentalmente o intenzionalmente, a terze parti non autorizzate.

Ciò potrebbe verificarsi quando gli operatori sanitari accedono ai dati sanitari da un dispositivo mobile o quando i pazienti archiviano i propri dati in una cartella clinica elettronica personale, poiché la perdita o il furto dei dispositivi su cui sono memorizzate informazioni riservate può costituire un serio problema di sicurezza. Al riguardo, data l'estrema delicatezza dei dati sanitari, è necessario che le applicazioni operanti nel campo della $m$ Health adottino soluzioni che offrano garanzie di sicurezza superiore specifiche e idonee, come la crittografia dei dati del paziente e meccanismi adeguati di autenticazione del paziente al fine di attenuare i rischi.

\section{Esempi di App utilizzate nel trattamento di alcune patologie croniche comuni}

Negli ultimi anni iniziano a essere presenti in letteratura svariati studi riguardanti l'utilizzo di App all'interno dell'ambito sanitario, valutando il modo in cui hanno modificato o influenzato tale contesto di applicazione. Attualmente, come già detto, gli sviluppatori privilegiano lo sviluppo di App ad uso "consumer" e quindi rivolte principalmente agli utenti comuni, piuttosto che ad uso professionale (rivolte, cioè, a un esperto/professionista del settore, quale il medico, ecc.). Ciò deriva dalla volontà di concepire l'applicazione come un software che non deve ricadere all'interno della regolamentazione a cui sono soggetti i dispositivi medici, a causa delle implicazioni e delle responsabilità che ne potrebbero derivare. Attualmente, la maggior parte delle App legate al mondo della sanità presenti all'interno dei negozi digitali svolge compiti di: diario alimentare, reminder per i farmaci, monitoraggio di alcuni parametri (tramite inserimento manuale dei dati), calcolatrici mediche e così via. Tali applicazioni trovano naturale collocazione all'interno dei percorsi terapeutici indirizzati ai pazienti che, per esempio, soffrono di patologie croniche quali nefropatie, diabete, ma- lattie cardiovascolari e obesità.

Gli studi analizzati si sono concentrati principalmente sul confrontare e valutare l'impatto e i benefici che tali applicazioni possono avere se utilizzate all'interno di un determinato percorso terapeutico, rispetto allo stesso percorso seguito "tradizionalmente".

\section{Le nefropatie croniche}

Nel mondo più di 60 milioni di individui sono affetti da patologie dell'apparato renale. La prevalenza delle nefropatie croniche è molto elevata e le stime mostrano un trend in crescita che aggraverà ulteriormente i costi che tali patologie hanno sui conti dell'assistenza sanitaria.

Nella gestione delle nefropatie croniche è necessario un grande impegno sia da parte del medico sia da parte del paziente, che dovrà attenersi scrupolosamente alle indicazioni fornitegli da parte dello specialista: dovrà innanzitutto modificare il proprio stile di vita e la propria alimentazione e dovrà seguire scrupolosamente le raccomandazioni del medico circa una corretta esecuzione della terapia.

Gli studi dimostrano che fornire una corretta informazione al paziente sulla propria patologia ha delle importanti ricadute sul piano dell'autogestione dei più importanti aspetti della condizione cronica e della compliance alla terapia; tuttavia si è anche potuto osservare che spesso i pazienti nefropatici non sono pienamente soddisfatti degli aspetti comunicativi nel rapporto medico-paziente e che talvolta non sono pienamente consapevoli dei risvolti clinici della propria patologia e di ciò che comporta vivere con una nefropatia cronica. D'altra parte, il paziente potrebbe non recepire correttamente le informazioni, anche se corrette ed esposte chiaramente dal medico, o recepirle solo parzialmente. L'utilizzo delle $A p p$, in questi casi, può essere un importante ausilio per il medico e soprattutto per il paziente, che potrebbe trovare in queste tecnologie un ulteriore strumento per la corretta gestione della propria patologia, soprattutto negli aspetti della vita quotidiana influenzati dalla malattia. A oggi, la maggior parte delle applicazioni mobili disponibili nell'ambito della nefrologia è dedicata specificatamente ai professionisti del settore o alla gestione di un corretto piano alimentare e alla motivazione del paziente. Eseguendo una ricerca nei principali negozi digitali di applicazioni per dispositivi mobili utilizzando chiavi di ricerca correlate alla nefropatia cronica (per esempio, kidney, CKD, nephrology, ecc.), si otterranno pochi risultati realmente significativi, tra i quali si rilevano principalmente applicazioni con funzioni di: calcolatrici mediche, diari alimentari, monitoraggio della funzione renale (tramite input dei dati dell'utente), Linee Guida ed e-learning.

\section{Le malattie cardiovascolari croniche}

Le malattie cardiovascolari sono una tra le principali cause di mortalità e morbilità in tutto il mondo, oltre a un'impor- 
tante voce nella spesa relativa all'assistenza sanitaria, sia in termini di ospedalizzazioni che in termini di prestazioni ambulatoriali e spesa farmaceutica, senza peraltro considerare la perdita di produttività lavorativa in una popolazione spesso ancora in età giovane.

I progressi raggiunti negli ultimi anni nell'ambito del trattamento degli eventi acuti hanno determinato un netto aumento della sopravvivenza dei pazienti e un netto aumento dei soggetti affetti da malattie cardiovascolari croniche.

Fattori importanti per la gestione e la prevenzione di queste malattie consistono nel controllo dei fattori di rischio cardiovascolari modificabili e quindi nel seguire uno stile di vita sano, nell'abolire il fumo, nel controllare il peso corporeo, nel praticare regolarmente attività fisica e così via.

Oltre a ciò, è importante per un cardiopatico cronico attenersi scrupolosamente alle indicazioni mediche anche per quanto riguarda la corretta assunzione della terapia farmacologica e il monitoraggio scrupoloso dei parametri vitali.

Le applicazioni mobili si stanno dimostrando un promettente strumento nell'aiuto dei pazienti nell'autogestione della malattia. Una metanalisi condotta analizzando i principali negozi digitali di $A p p$ ha evidenziato che le App per le malattie cardiovascolari più popolari sono i monitor cardiaci e le calcolatrici mediche. Altre categorie di App, maggiormente indirizzate all'uso professionale, includono il monitoraggio della pressione, la formazione specialistica, la lettura, l'interpretazione, la refertazione dell'ECG registrato tramite specifici sensori/holter (dispositivi medici) e le istruzioni per la CPR.

\section{La gestione del diabete}

Il diabete è una patologia cronica che richiede un controllo costante e continuativo e i pazienti devono recarsi regolarmente presso gli ambulatori medici solo per monitorare i parametri essenziali per la corretta gestione della malattia.

Sono disponibili diverse applicazioni mobili per aiutare i pazienti nel controllo dei valori della glicemia e nell'impostazione di una dieta equilibrata e di una corretta attività fisica, consentendo alle persone affette da questa patologia di avere un migliore controllo del proprio stato di salute. Queste applicazioni aiutano anche a ridurre il numero di volte che i pazienti devono visitare i centri diabetologici dimostrando così di essere una soluzione conveniente per la gestione del diabete. Tali funzionalità supportano il medico nell'educazione del paziente alla corretta autogestione della malattia e forniscono il supporto per ridurre il rischio di disabilità a lungo termine causate dalla cattiva gestione della condizione patologica.

Le funzionalità principali offerte da tali applicazioni includono il monitoraggio della glicemia, avvisi per l'assunzione di farmaci, la gestione della dieta, il monitoraggio dell'esercizio fisico e così via, mentre le funzioni secondarie rientrano nell'ambito della formazione, del supporto decisionale, del monitoraggio del peso/BMI, della comunicazione, del social networking e così via.

Nel 2010 è stato condotto uno studio su un gruppo selezionato di pazienti attraverso questionari e interviste e si è osservato che le applicazioni mobili per la gestione del diabete hanno un impatto positivo sui fruitori. Tale impatto si è tradotto nella motivazione degli utenti nel seguire una dieta sana e un regime di esercizio fisico regolare, mantenendo valori di glicemia normali con un conseguente miglioramento dello stato di salute. Nel loro studio, gli Autori hanno anche notato alcune lacune delle App analizzate. Per esempio, è emerso che molte delle applicazioni di autogestione del diabete non includono informazioni di carattere educativo, anche se le Linee Guida cliniche sottolineano l'importanza dell'educazione del paziente. Un altro elemento importante che gli Autori hanno rilevato è la mancanza del collegamento di tali applicazioni con la cartella sanitaria personale.

\section{La gestione del peso}

Obesità e sovrappeso sono diventati un problema diffuso in tutto il mondo. L'obesità è una patologia che può portare a molte condizioni gravi come il diabete di tipo 2, malattie cardiache, malattie osteoarticolari, sindrome delle apnee ostruttive del sonno, sindrome metabolica e così via.

Il trattamento dell'obesità può essere complesso in quanto l'eziopatogenesi di questa patologia è multifattoriale e la risposta al trattamento stesso può variare da paziente a paziente. Metodi generali utilizzati per la riduzione del peso e la gestione di questa condizione includono un intervento sullo stile di vita con riduzione dell'apporto calorico, attività fisica, supporto psicologico e così via.

La tecnologia mobile può essere utile nel fornire spunti necessari per un corretto stile di vita e per il cambio delle abitudini comportamentali necessarie per il controllo del peso. Uno studio effettuato ha preso in considerazione le applicazioni di gestione del peso disponibili sui principali negozi digitali $A p p$ ed è emerso che le funzionalità fornite da queste applicazioni vanno dal monitoraggio della dieta e del peso corporeo al diario alimentare e a norme per una cucina salutare. Ci sono molte strategie basate sulla teoria dell'educazione comportamentale che possono essere impiegate per aumentare l'efficacia degli interventi per la riduzione e la gestione del peso, come l'automonitoraggio, l'impostazione di un obiettivo realistico, l'auto-ricompensa e così via.

\section{Conclusioni}

Le App legate al mondo della sanità digitale sono in rapida crescita, sia come numero che come popolarità, con ricadute positive sia per i professionisti del settore che per gli utenti (spesso pazienti). Ciò può portare a una vera e propria rivoluzione del modo in cui si può usufruire della sanità. II mercato legato alla mHealth rappresenta un importante fattore di spinta sui Competitor operanti nel mondo del medicale ma 
contemporaneamente è fondamentale un rapido miglioramento nella regolamentazione a tutela degli utenti (pazienti).

A oggi è possibile osservare come le App pubblicate nei principali negozi digitali siano abbastanza controllate in merito alla presenza di codice malevolo e di autorizzazione all'accesso dei dati personali degli utenti. È anche vero, però, che i loro Gestori stanno mostrando un forte interesse a entrare nel mercato della salute, ma non a vendere "dispositivi medici". La differenza, come già detto, risiede nel fatto che, nel secondo caso, i Gestori dovrebbero imporre agli sviluppatori di accettare norme e condizioni molto rigorose nell'ambito del territorio nel quale le $A p p$ vengono prodotte e successivamente distribuite, sottoponendoli a procedure lunghe e costose, con relative ricadute in ordine alla responsabilità giuridica. La tendenza, quindi, è quella di aggirare il problema, presentando $\mathrm{i}$ prodotti, le $A p p$, come semplici modalità di controllo del benessere degli utenti, anche se, in realtà, spesso, si tratta di veri e propri dispositivi medici, portando a un'ambiguità nell'offerta delle applicazioni sulla salute.

Inoltre, per le App legate alla sanità, proprio perché più vicine al mondo "ICT" piuttosto che a quello dei dispositivi medici, ciò che tende a prevalere rispetto alla valutazione di esperti di settore è il parere delle persone che le hanno scaricate ed utilizzate: in questo senso, quindi, ciò che induce gli utenti ad installare o meno una App non è tanto la sua validazione scientifica quanto, piuttosto, il tasso di gradimento in rete espresso dai consumatori che le hanno già scaricate. Quindi, in totale opposizione rispetto al processo di validazione di un dispositivo medico, soggetto a una regolamentazione molto precisa, spesso emergono la mancanza o la carenza di un'adeguata validazione scientifica delle applicazioni per la sicurezza e per l'efficacia proprio perché non vi è un requisito normativo per la sperimentazione di $A p p$ non classificabili come dispositivi. In conclusione, sarebbe auspicabile che gli sviluppatori, nel momento in cui propongono un prototipo, lo sottopongano a una validazione $o$ a una valutazione dei rischi e dei benefici, proprio come avviene per i dispositivi medici, poiché anche le informazioni generali riferite alla salute e al benessere devono essere accurate e affidabili, perché su di esse si potrebbero basare decisioni critiche rispetto alla salute dell'utilizzatore, considerabile, quindi, a tutti gli effetti un paziente.

\section{Disclosures}

Financial support: No financial support was received for this submission.

Conflict of interest: The authors have no conflict of interest.

\section{Bibliografia}

1. http://www.cisco.com/c/en/us/solutions/collateral/serviceprovider/visual-networking-index-vni/mobile-white-paperc11-520862.htm

2. http://www.imedicalapps.com/2015/09/ims-health-apps-re port/

3. Medical Device Directive $2007 / 47 /$ EEC

4. http://meddev.info/

5. http://meddev.info/wp-content/uploads/2016/08/MEDDEV_2_1_6.pdf

6. http://ec.europa.eu/DocsRoom/documents/12867/attachments/1/translations/en/renditions/native

7. http://ec.europa.eu/justice/data-protection/article-29/documentation/opinion-recommendation/files/2014/wp223_ en.pdf

8. https://www.ft.com/content/b709cf4a-12dd-11e3-a05e00144 feabdc0

9. Becker S, Becker S, Kribben A, et al. User profiles of a smartphone application to support drug adherence experiences from the iNephro project. PLoS One 2013;8(10):e78547.

10. Diamantidis CJ, Becker S. Health information technology (IT) to improve the care of patients with chronic kidney disease (CKD). BMC Nephrol 2014;15:7.

11. https://www.infoway-inforoute.ca/en/what-we-do/newsevents/newsroom/2016-news-releases/7201-study-evaluatesmobile-app-for-chronic-kidney-disease-patients

12. Martínez-Pérez B, de la Torre-Díez I, López-Coronado M, Herreros-González J. Mobile Apps in Cardiology: Review. JMIRMhealth Uhealth 2013;1(2):e15.

13. Seto E, Leonard KJ, Cafazzo JA, Barnsley J, Masino C, Ross HJ. Mobile phone-based telemonitoring for heart failure management: a randomized controlled trial. J Med Internet Res 2012;14(1):e31.

14. Arsand E, Tatara N, $\emptyset$ stengen G, Hartvigsen G. Mobile PhoneBased Self-Management Tools for Type 2 Diabetes: The FewTouch Application. J Diabetes Sci Technol 2010;4(2):328-336.

15. El-Gayar O, Timsina P, Nawar N, Eid W. Mobile applications fordiabetes self-management:status and potential. J Diabetes Sci Technol 2013;7(1):247-262.

16. Ryan A, Ristau BS, Yang J, et al, Evaluation and Evolution of Diabetes Mobile Applications: Key Factors for Health Care Professionals Seeking to Guide Patients. PharmD Diabetes Spectrum 2013;26(4):211-215.

17. Azar KM, Lesser LI, Laing BY, et al. Mobile applications forweight management: theory-based content analysis. Am J Prev Med 2013;45(5):583-589.

18. In Vitro Diagnostic Medical Device Directive 98/79/EEC

19. http://ec.europa.eu/dgs/health_food-safety/index_it.htm

20. http://www.patient-view.com/-bull-directories.html

21. http://www.mobimed.it/

22. http://medicapp.info/appmediche/

23. http://www.fda.gov/downloads/medicaldevices/deviceregulationandguidance/guidancedocuments/ucm263366.pdf

24. The mobile health global market report 2013-2017: the commercialisation of mHealth apps. Research2Guidance. 2013;(Vol. 3).

25. Sviluppo di dispositivi medici software. Ken Halle, Triteq, Stype, UK, 01/Jun/2010. 